\title{
ON EULER TRANSFORMS
}

\section{PHILIP HARTMAN AND AUREL WINTNER}

Let $E_{0}$ denote the class of non-constant functions satisfying

$$
d \phi(x) \geqq 0 \quad \text { where } 0 \leqq x<\infty .
$$

Then the Euler transform

$$
\phi_{\lambda}(x)=\int_{0}^{\infty}(x+t)^{-\lambda} d \phi(t), \quad \text { where } 0<x<\infty,
$$

is defined for some $\phi$ of class $E_{0}$. Let $E_{\lambda}$ denote the class of all functions $\phi_{\lambda}$ belonging to a fixed $\lambda>0$ and to some $\phi$ of class $E_{0}$. It will be shown that

$$
E_{\lambda} \text { is a (proper) subset of } E_{\mu} \text { if } \lambda<\mu .
$$

This implies that

$$
E_{\infty}=\lim _{\lambda \rightarrow \infty} E_{\lambda} \text { if } E_{\infty}=\sum_{0<\lambda<\infty} E_{\lambda} .
$$

The class $E_{\infty}$ is closely related to the Hausdorff-Bernstein class, consisting of all functions which are completely monotone for $0<x$ $<\infty$. Let $E^{\infty}$ denote the latter class. It will be shown that

$$
E_{\infty} \text { is a (proper) subset of } E^{\infty}
$$

and that, with reference to the "natural" topology on $E^{\infty}$,

$$
E_{\infty} \text { is dense on } E^{\infty} \text {. }
$$

It should be noted that $E^{\infty}$ consists of all functions representable in the form

$$
\phi^{\infty}(x)=\int_{0}^{\infty} e^{-x t} d \phi(t), \quad \text { where } 0<x<\infty,
$$

provided that $\phi$, instead of being subject to both restrictions (1), is subject only to the second of those restrictions and to the assumption that the integral ( 7$)$ is convergent at every $x>0$ (but not necessarily at $x=0)$. By the "natural" topology on $E^{\infty}$ is meant that defined by the Helly convergence of monotone functions.

Proof of (3). It is readily verified from (1) and (2) that, as $x \rightarrow \infty$, no $\phi_{\lambda}(x)$ can tend to 0 as strongly as $x^{-\mu}$, if $\mu>\lambda$. On the

Received by the editors January 31, 1949. 
other hand, (2) shows that $\phi_{\mu}(x)=x^{-\mu}$ if $\phi(t)=\operatorname{sgn} t$. Hence, the parenthetical assertion of (3) will need no further proof.

The main assertion of (3) is that, if $0<\lambda<\mu$, there belongs to every $\phi_{\lambda}(t)$ of class $E_{\lambda}$ some $\phi^{*}(t)$ of class $E_{0}$ satisfying

$$
\phi_{\lambda}(x)=\phi_{\mu}^{*}(x), \quad \text { where } 0<x<\infty .
$$

It will be shown that such a $\phi^{*}(t)$ is supplied by the absolutely continuous function having the derivative

(9) $d \phi^{*}(t) / d t=\mathrm{A} \int_{0}^{t}\left(t-{ }^{-}\right)^{\mu-\lambda-1} d \phi(s)\left(0<t<\infty, \phi^{*}(0)=\phi^{*}(+0)\right)$, where $\mathbf{A}=\mathbf{A}(\lambda, \mu)$ is a positive constant.

In view of (2), the assertion of (8) and (9) means that

$$
\int_{0}^{\infty}(x+t)^{-\lambda} d \phi(t)=\mathrm{A} \int_{0}^{\infty}(x+t)^{-\mu}\left\{\int_{0}^{t}(t-s)^{\mu-\lambda-1} d \phi(s)\right\} d t,
$$

where $0<x<\infty$. It follows therefore from (1), and from (the Stieltjes form of) Fubini's theorem, that it is sufficient to verify the identity

$$
\int_{0}^{\infty}(x+t)^{-\lambda} d \phi(t)=\mathrm{A} \int_{0}^{\infty}\left\{\int_{0}^{\infty}(x+t)^{-\mu}(t-s)^{\mu-\lambda-1} d t\right\} d \phi(s) .
$$

But the latter holds for every $\phi$ of class $E_{0}$ if

$$
(x+t)^{-\lambda}=\mathrm{A} \int_{t}^{\infty}(x+s)^{-\mu}(s-t)^{\mu-\lambda-1} d s
$$

is an identity in $(x, t)$, where $x>0, t>0$. Hence, if the integration variable $s$ is replaced by $s-t$, and if $x+t$ is then called $x$, it follows that it is sufficient to verify the identity

$$
x^{-\lambda}=\mathrm{A} \int_{0}^{\infty}(x+s)^{-\mu_{s} \mu-\lambda-1} d s,
$$

where $\mathbf{A}$ is independent of $x$. Finally, the truth of this identity follows by changing $s$ to $x s$ (at a fixed $x>0$ ).

This proves (3). It also follows that the value of the constant $\mathbf{A}=\mathbf{A}(\lambda, \mu)$ which occurs in (9) is given by

$$
1=\mathrm{A} \int_{0}^{\infty}(1+s)^{-\mu_{s}^{\mu-\lambda-1}} d s .
$$

Incidentally, the last integral can readily be transformed into the 
integral defining $B(\lambda, \mu)=\Gamma(\lambda) \Gamma(\mu-\lambda) / \Gamma(\mu)$; so that, in (9),

$$
A=A(\lambda, \mu)=1 / B(\lambda, \mu) .
$$

Proof of (5). It is seen from (1) that $(-1)^{n}$ times the $n$th derivative of the function (2) is non-negative for $n=0,1,2, \ldots$. This means that every class $E_{\lambda}$ is contained of the Hausdorff-Bernstein class, $E^{\infty}$. Hence, by (4), $E_{\infty}$ is contained in $E^{\infty}$. The parenthetical part of (5) follows from the fact that $e^{-x}$ is in $E^{\infty}$, but $e^{-x}$ is not in $E_{\infty}$. For otherwise $e^{-x}$ would be in $E_{\lambda}$ some $\lambda$, which would imply, for $x>0$ and $t_{0}>0$, that

$$
e^{-x} \geqq\left(x+t_{0}\right)^{-\lambda}\left(\phi\left(t_{0}\right)-\phi(0)\right),
$$

by (1) and (2). If $t_{0}$ is chosen so that $\phi\left(t_{0}\right)-\phi(0)>0$, the last formula line leads to a contradiction for large $x$. This contradiction shows that $e^{-x}$ cannot be in $E_{\infty}$ and completes the proof of (5).

Proof of (6). Let $b>0$ and $\lambda>0$. Then it is readily verified that

$$
b^{\lambda} \int_{0}^{\infty}(x+t)^{-\lambda} d \operatorname{sgn}(t-b)=(1+x / b)^{-\lambda},
$$

where $0 \leqq x<\infty$. Clearly, the expression on the left of this identity represents a function of class $E_{\lambda}$. On the other hand, the expression on the right tends to $e^{-a x}$ if $\lambda \rightarrow \infty$ and $b=\lambda / a$, where $a$ is any positive constant. Accordingly, every function of the form $e^{-a x}$ is a limit, as $\lambda \rightarrow \infty$, of functions contained in $E_{\lambda}$. Hence, the same is true of every function of the form

$$
\sum_{k=1}^{m} c_{k} e^{-a_{k} x}
$$

where $c_{k}, a_{k}$ are arbitrary non-negative constants. Since the latter sum is identical with the case

$$
\phi(x)=\sum_{a_{k} \leq x} c_{k}
$$

of the transform (7), the assertion of (6) now follows by a standard application of Helly's theorems on monotone functions.

The JoHNS Hopkins UNIVERSITY 